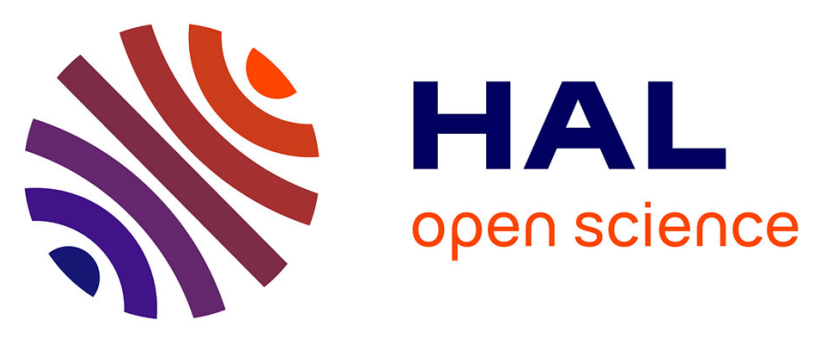

\title{
Robust coherent spin centers from stable azafullerene radicals entrapped in cycloparaphenylene rings
}

\author{
Yuri Tanuma, Anastasios Stergiou, Andreja Bužan B Bobnar, Mattia \\ Gaboardi, Jeremy Rio, Jannis Volkmann, Hermann A. Wegner, Nikos \\ Tagmatarchis, Christopher P. Ewels, Denis Arčon
}

\section{To cite this version:}

Yuri Tanuma, Anastasios Stergiou, Andreja Bužan B Bobnar, Mattia Gaboardi, Jeremy Rio, et al.. Robust coherent spin centers from stable azafullerene radicals entrapped in cycloparaphenylene rings. Nanoscale, In press, 10.1039/D1NR06393F . hal-03429540

\section{HAL Id: hal-03429540 https://hal.science/hal-03429540}

Submitted on 15 Nov 2021

HAL is a multi-disciplinary open access archive for the deposit and dissemination of scientific research documents, whether they are published or not. The documents may come from teaching and research institutions in France or abroad, or from public or private research centers.
L'archive ouverte pluridisciplinaire HAL, est destinée au dépôt et à la diffusion de documents scientifiques de niveau recherche, publiés ou non, émanant des établissements d'enseignement et de recherche français ou étrangers, des laboratoires publics ou privés. 


\section{Robust coherent spin centers from stable azafullerene radicals entrapped in cycloparaphenylene rings}

Yuri Tanuma, Anastasios Stergiou, Andreja Bužan Bobnar, Mattia Gaboardi, Jeremy Rio, Jannis Volkmann, Hermann A. Wegner, Nikos Tagmatarchis, Christopher P. Ewels, * Denis Arčon*

Y. Tanuma

Institut des Matériaux Jean Rouxel, CNRS / Université de Nantes, BP32229, 44322 Nantes, France

Graduate School of Interdisciplinary New Science, Toyo University, 2100 Kujirai, Kawagoe, 350-8585, Saitama, Japan

Dr. A. Stergiou, Prof. N. Tagmatarchis

Theoretical and Physical Chemistry Institute, National Hellenic Research Foundation, 48 Vassileos Constantinou Avenue, 11635 Athens, Greece

A. B. Bobnar

Jožef Stefan Institute, Jamova 39, 1000 Ljubljana, Slovenia

Dr. M. Gaboardi

Sincrotrone Trieste S.C.p.A., Strada Statale 14 Km 163.5, I-34149 Trieste, Italy

Jannis Volkmann, Prof. Dr. H. A. Wegner

Institute of Organic Chemistry, Justus Liebig University Giessen, Heinrich-Buff-Ring 17, 35392, Giessen, Germany

Center for Materials Research (LaMa), Justus Liebig University Giessen, Heinrich-Buff-Ring 16, 35392, Giessen, Germany

Dr J. Rio, Dr. C. P. Ewels

Institut des Matériaux Jean Rouxel, CNRS / Université de Nantes, BP32229, 44322 Nantes, France

E-mail: chris.ewels@cnrs-imn.fr

Prof. D. Arčon

Jožef Stefan Institute, Jamova 39, 1000 Ljubljana, Slovenia.

Faculty of Mathematics and Physics, University of Ljubljana, Jadranska 19, 1000 Ljubljana, Slovenia.

E-mail: denis.arcon@ijs.si

Keywords: cycloparaphenylenes, azafullerenes, EPR, qubits

Molecular entities with robust spin-1/2 are natural two-level quantum systems for realizing qubits and are key ingredients of emerging quantum technologies such as quantum computing. Here we show that robust and abundant spin-1/2 species can be created in-situ in the solid state from spin-active azafullerene $\left(\mathrm{C}_{59} \mathrm{~N}\right)$ cages supramolecularly hosted in crystals of [10]cycloparaphenylene ([10]CPP) nanohoops. This is achieved via a two-stage thermallyassisted homolysis of the parent diamagnetic [10]CPP $\supset\left(\mathrm{C}_{59} \mathrm{~N}\right)_{2} \subset[10] \mathrm{CPP}$ supramolecular 
complex. Upon cooling, the otherwise unstable $\mathrm{C}_{59} \mathrm{~N}^{\bullet}$ radical is remarkably persistent with a measured radical lifetime of several years. Additionally, pulsed electron paramagnetic resonance measurements show long coherence times, fulfilling a basic condition for any qubit manipulation, and observed Rabi oscillations demonstrate single qubit operation. These findings together with rapid recent advances on the synthesis of carbon nanohoops offer the potential to fabricate tailored cycloparaphenylene networks hosting $\mathrm{C}_{59} \mathrm{~N}^{\bullet}$ centers, providing a promising platform for building complex qubit circuits.

\section{Introduction}

Discrete energy levels of quantum spins at the molecular level are a promising platform for realising qubits. ${ }^{1}$ The major challenge, despite enormous progress in the past decade, remains how to build complex circuits from such molecular spin entities with sufficiently long coherence times in order to be integrated into quantum device and to, for example, conduct quantum computation algorithms. In most cases, molecular structures containing transition metal ions with $d$-electrons or lanthanides with $f$-electrons were studied. While various ingenious approaches were developed to prolong coherence times, ${ }^{2-4}$ building circuits still remains difficult. Fullerenes, well known for their $\pi$-electron molecular magnetism and superconductivity, ${ }^{5-7}$ represent a potentially interesting metal-free molecular spin alternative. Fullerene-based qubits with relatively long coherence times were indeed used to store quantum information, as shown in several electron paramagnetic resonance (EPR) experiments. ${ }^{8-10}$ The focus so far has been on the endohedral molecule N@C60, which has electronic spin of $S=3 / 2$ as in atomic nitrogen, and isotropic hyperfine interaction to nitrogen nuclear $\operatorname{spin} A\left({ }^{14} \mathrm{~N}\right)=15.73$ MHz. ${ }^{11}$ Employing the standard methods of pulsed EPR it is possible to initialize a given spin state of $\mathrm{N} @ \mathrm{C}_{60}$, and the protected environment inside $\mathrm{C}_{60}$ is responsible for the exceptionally long electron-spin dephasing time $T_{2}=210 \mu$ s measured in frozen $\mathrm{CS}_{2}$ solution at $-83^{\circ} \mathrm{C} .^{12}$ While these data satisfy the criterion of long coherence times, $\mathrm{N} @ \mathrm{C}_{60}$ fails to provide a practical path to build complex circuits, simply because the yield for preparing $\mathrm{N} @ \mathrm{C}_{60}$ is too low typically the concentration of the paramagnetic centers produced by ${ }^{14} \mathrm{~N}$-bombardment of $\mathrm{C}_{60}$ is only in the order of $10^{-7}-10^{-4}$ per $\mathrm{C}_{60}$ molecule. Although $\mathrm{N} @ \mathrm{C}_{60}$-enriched samples can be isolated by exhausting separation assays, with the aid of preparative high-performance liquid chromatography (HPLC), ${ }^{13} \mathrm{~N} @ \mathrm{C}_{60}$ remains an expensive exotic qubit platform. Additionally, it has been established that the encapsulated nitrogen atom escapes the cage in the temperature range of $125-225^{\circ} \mathrm{C} .{ }^{14}$ 
An alternative spin-active candidate for the fabrication of robust qubit-arrays could be azafullerene $\mathrm{C}_{59} \mathrm{~N}$, where one nitrogen replaces a carbon atom on the fullerene skeleton. Due to valence difference between $\mathrm{N}$ and $\mathrm{C}, \mathrm{C}_{59} \mathrm{~N}$ is a closed-cage heterofullerene radical where the odd electron resides on the carbon atom neighbouring the embedded nitrogen. ${ }^{15}$ These spinactive species can be directly accessed by thermolysis or photolysis of the parent diamagnetic azafullerene dimer $\left(\mathrm{C}_{59} \mathrm{~N}\right)_{2} \cdot{ }^{16-18}$ Further, the azafullerenyl $\mathrm{C}_{59} \mathrm{~N}^{\bullet}$ radical centers can also be generated by the thermolysis of the non-magnetic hydroazafullerene $\mathrm{C}_{59} \mathrm{HN} .{ }^{19,20}$ The dimer $\left(\mathrm{C}_{59} \mathrm{~N}\right)_{2}$ is synthesized via a conventional chemical route in efficient macroscopic yields, i.e. $10 \%$ with respect to $C_{60}$, and requires only a simple purification by column chromatography, constituting a versatile candidate for generating the paramagnetic $\mathrm{C}_{59} \mathrm{~N}^{\bullet}$ centers. To date, the integration of $\mathrm{C}_{59} \mathrm{~N}^{\bullet}$ radical centers into spin-active arrays has not been explored because of their high reactivity to ultra-rapid dimerization (under inert conditions) and oxidation (in ambient environment). Therefore, protection of the paramagnetic $\mathrm{C}_{59} \mathrm{~N}^{\bullet}$ from dimerization back to diamagnetic $\left(\mathrm{C}_{59} \mathrm{~N}\right)_{2}$ or oxidation to $\mathrm{C}_{59} \mathrm{~N}^{+}$and $\mathrm{C}_{59} \mathrm{NO}$ species is very challenging, but highly desirable.

Recently, we developed a supramolecular complex in order to shield the labile azafullerenyl radical. Specifically, we took advantage of the supramolecular concave-convex $\pi$ - $\pi$ interactions between a carbon nanobelt, [10]cycloparaphenylene ([10]CPP), and the azafullerene $\left(\mathrm{C}_{59} \mathrm{~N}\right)$ cage (Figure 1a), enabling the formation of $\mathrm{C}_{59} \mathrm{~N}^{*} \subset[10] \mathrm{CPP}$ radical species in solution, i.e., of 1-chloronaphthalene. ${ }^{21}$ The spin-active supramolecular complex was generated by photolysis (532 nm) of the parent non-magnetic [10]CPP $\supset\left(\mathrm{C}_{59} \mathrm{~N}\right)_{2} \subset[10] \mathrm{CPP}$ complex with increased efficiency of the photolysis by a factor of 300. Although this demonstrates promise for bottomup fabrication of nanostructured spin-active arrays of $\mathrm{C}_{59} \mathrm{~N}^{*}$, the result was liquid-phase based and requires optical activation, and as such is not appropriate for creating practical surface qubit arrays.
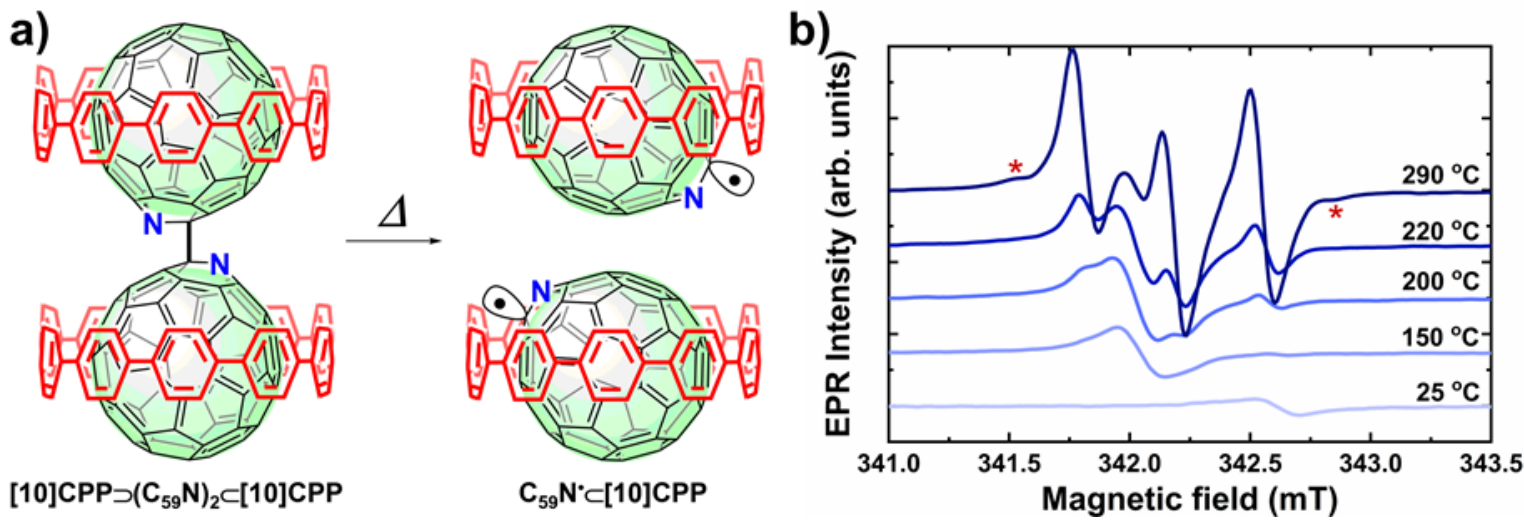
Figure 1. Formation of $\mathrm{C}_{59} \mathrm{~N}$ radicals from stable dimers $\left(\mathrm{C}_{59} \mathrm{~N}\right)_{2}$ by heating. (a) Schematic structure of [10]CPP $\supset\left(\mathrm{C}_{59} \mathrm{~N}\right)_{2} \subset[10] \mathrm{CPP}$ non-magnetic unit, which yields $\mathrm{C}_{59} \mathrm{~N}^{*} \subset[10] \mathrm{CPP}$ radical species upon breaking the $\mathrm{C}-\mathrm{C}$ bond. (b) The evolution of X-band EPR spectra of asprepared [10]CPP $\supset\left(\mathrm{C}_{59} \mathrm{~N}\right)_{2} \subset[10] \mathrm{CPP}$ powder upon heating the sample from room temperature to $290{ }^{\circ} \mathrm{C}$.

Encouraged by the previous solid-state EPR characterization of $\left(\mathrm{C}_{59} \mathrm{~N}\right)_{2}$ and $\mathrm{C}_{59} \mathrm{HN}$, which demonstrated the ease of generating $\mathrm{C}_{59} \mathrm{~N}^{*}$ radicals via instead a thermolysis route ${ }^{18,20}$ we herein report on the generation of long-term stable $\mathrm{C}_{59} \mathrm{~N}^{*}$ radical centers entrapped in [10]CPP in the solid state. The objective is two-fold, namely, to generate $\mathrm{C}_{59} \mathrm{~N}^{*} \subset[10] \mathrm{CPP}$ radicals via the thermally-induced homolysis of the parent diamagnetic $[10] \mathrm{CPP} \supset\left(\mathrm{C}_{59} \mathrm{~N}\right)_{2} \subset[10] \mathrm{CPP}$ supramolecular complex, and to handle and assess the generated $\mathrm{C}_{59} \mathrm{~N}^{\circ} \subset[10] \mathrm{CPP}$ radical species in the absence of any solvent as wetting media. During microwave-assisted thermal annealing of the diamagnetic [10]CPP $\supset\left(\mathrm{C}_{59} \mathrm{~N}\right)_{2} \subset[10] \mathrm{CPP}$ powder, a two-stage process is identified and discussed: 1) at $\sim 150{ }^{\circ} \mathrm{C}$ the dimer C-C bond is weakened, allowing [10]CPPencircled azafullerenes to rotate and bind to neighbouring [10]CPP $\supset\left(\mathrm{C}_{59} \mathrm{~N}\right)_{2} \subset[10] \mathrm{CPP}$, furnishing spin-active species with $S=1 / 2$, and 2) above $\sim 200^{\circ} \mathrm{C}$ where the latter intermediate species are thermally dismantled and release $C_{59} \mathrm{~N}^{\bullet} \subset[10] \mathrm{CPP}$ radicals with $S=1 / 2$, which emerge and dominate the EPR spectra (Figure 1). Incorporation of the [10]CPP nanobelts around the azafullerene cages benefits the overall process in two ways, a) enabling much higher concentrations of $\mathrm{C}_{59} \mathrm{~N}^{*} \subset[10] \mathrm{CPP}$ at lower temperature, as compared to the corresponding azafullerenyl radical species generated from $\left(\mathrm{C}_{59} \mathrm{~N}\right)_{2}$ in the absence of [10]CPP, and b) at ambient conditions the radical signal is remarkably stable and persistent for more than 30 months. $\mathrm{C}_{59} \mathrm{~N}^{\bullet}$ shows a surprisingly long room-temperature spin coherence lifetime and can be addressed as a qubit, with many Rabi oscillations demonstrated. The present study thus demonstrates the surprising long-term stability of $\mathrm{C}_{59} \mathrm{~N}^{\bullet}$ radicals at the solid-state, when entrapped by [10]CPP nanobelts. Such robust spin centres are key ingredients for emerging quantum technology. They pave the way to bottom-up building of localised $\mathrm{C}_{59} \mathrm{~N}^{\bullet}$ qubit circuits, which may be "wired" through the spin-redistributed states. The emerging functionalities of such materials will thus likely benefit from such wavefunction duality.

\section{Results}

\subsection{EPR studies on $[10] C P P-C_{59} N$ complexes}


EPR has shown itself to be an extremely valuable and sensitive technique to detect the formation of $\mathrm{C}_{59} \mathrm{~N}^{*} \subset[10] \mathrm{CPP}$ radicals in 1-chloronaphthalene upon photolysis. ${ }^{21}$ Here we employ a similar strategy and probe the thermal breaking of $\left(\mathrm{C}_{59} \mathrm{~N}\right)_{2}$ dimer bond instead in the solid. Briefly, [10]CPP $\supset\left(\mathrm{C}_{59} \mathrm{~N}\right)_{2} \subset[10] \mathrm{CPP}$ solid is prepared by adding a 2-fold molar excess [10]CPP solution in 1-chloronaphthalene in a solution of $\left(\mathrm{C}_{59} \mathrm{~N}\right)_{2}$ in the same medium. The mixture was stirred for $24 \mathrm{~h}$ at room temperature to reach a complexation equilibrium, and [10]CPP $\supset\left(\mathrm{C}_{59} \mathrm{~N}\right)_{2} \subset[10] \mathrm{CPP}$ precipitated out upon refrigeration of the mixture, washed with hexane and dried under vacuum. While it is not possible to fully determine the crystalline structure (see Supporting Information and Figure S1 for powder synchrotron X-ray diffraction characterization), FT-IR and Raman spectroscopy (Figure S2 and the related text in Supporting Information) as well as NMR (see Figure S3 for ${ }^{13} \mathrm{C}$ and $1 \mathrm{H}$ solid-state magic angle spinning NMR data and discussion) and EPR spectroscopies (below) demonstrate clearly the molecular interaction between the [10]CPP nanobelts and the encapsulated $\mathrm{C}_{59} \mathrm{~N}$. The as-prepared [10]CPP $\supset\left(\mathrm{C}_{59} \mathrm{~N}\right)_{2} \subset[10] \mathrm{CPP}$ powder shows a very weak X-band EPR signal at $g=1.9988$ (Figure 1b), which has previously been frequently associated with a well-known $\mathrm{C}_{60}{ }^{-}$signal, arising from a small concentration of defective fullerene impurities diluted in an otherwise EPR silent diamagnetic sample. ${ }^{22}$ However, in the current study, the presence of even minute amounts of $\mathrm{C}_{60}$ as impurity within $\left(\mathrm{C}_{59} \mathrm{~N}\right)_{2}$ has been precluded as a result of HPLC purification, and this signal must therefore come from another source (HPLC trace shown in Supplementary Materials).

Upon heating from room temperature, the $g=1.9988$ signal gradually disappears and at around $\sim 100^{\circ} \mathrm{C}$, a new much stronger signal starts to grow at a nearly free-electron value of $g=2.0022$ (Figure 1b). This signal has a Lorentzian lineshape with a linewidth of $\Delta \boldsymbol{B}_{\mathbf{1} / \mathbf{2}}=\mathbf{0 . 3 5} \mathrm{mT}$ and shows no fine structure due to hyperfine coupling to ${ }^{1} \mathrm{H}$ or ${ }^{14} \mathrm{~N}$ nuclear moments. The absence of sizeable hyperfine coupling has been further confirmed with pulsed EPR using the electron spin echo envelope modulation (ESEEM) technique, which shows only a simple exponential decay of the echo signal without characteristic hyperfine modulations (Figure S4). The absence of ${ }^{1} \mathrm{H}$ and ${ }^{14} \mathrm{~N}$ hyperfine coupling and the typical $g$-factor value suggest that this signal is due to spatially extended spin states present in powder samples, but not in 1-chloronaphthalene solution where no such signal was observed upon $532 \mathrm{~nm}$ laser-photolysis (Figure S5). The absence of ${ }^{1} \mathrm{H}$ ESEEM signal also rules out the possibility that this signal arises from unpaired charge localized on the [10]CPP, or due to a reaction between $\mathrm{C}_{59} \mathrm{~N}^{\bullet}$ and [10]CPP (Figure S6 and Supporting Information for density functional theory (DFT) calculations of such possibility), and thus the signal is still due to an azafullerenyl radical. The absence of ${ }^{14} \mathrm{~N}$ 
ESEEM signal further confines these paramagnetic centers to carbons of $\mathrm{C}_{59} \mathrm{~N}$ entities distant from the $\mathrm{N}$ atom of $\mathrm{C}_{59} \mathrm{~N}$. In this context, we note its close resemblance to EPR signals observed in $\mathrm{C}_{59} \mathrm{HN}$ or in $\mathrm{C}_{59} \mathrm{~N}$ doped $\mathrm{C}_{60}$ powders. ${ }^{\mathbf{1 8 , 2 0 , 2 3}}$ Such EPR active states were in these cases promoted by the formation of intermediate $\mathrm{C}_{59} \mathrm{~N}-\mathrm{C}_{59} \mathrm{HN} \mathrm{N}^{\bullet}$ and $\mathrm{C}_{59} \mathrm{~N}-\mathrm{C}_{60}{ }^{\bullet}$ complexes, respectively, accompanied with spin redistribution over the surface of the $\mathrm{C}_{59} \mathrm{HN}$ or $\mathrm{C}_{60}$ and a corresponding shift of spin density away from the nitrogen atom of the $\mathrm{C}_{59} \mathrm{~N}$.

The concentration of these $g=2.0022$ intermediate temperature spin species continues to grow upon heating. However, close inspection of the spectrum taken at $T=150{ }^{\circ} \mathrm{C}$ (Figure 1b) already indicates small deviations from the ideal Lorentzian lineshape due to the presence of a further signal, which becomes increasingly apparent at higher temperature and begins to dominate the EPR spectra above $220^{\circ} \mathrm{C}$. At the same time, the intensity of the $g=2.0022$ signal starts to slightly decrease, which implies that the two processes are intimately linked and take place on the azafullerene cage. This high-temperature signal is characterised by a triplet of sharp peaks, which are split by $0.36 \mathrm{mT}$ and are centered at $g=2.0014$. As this signal has been observed before in $\mathrm{C}_{59} \mathrm{~N}^{\bullet} \subset[10] \mathrm{CPP}$ radical species generated upon photolysis in 1-chloronaphthalene, ${ }^{21}$ we assign it to the well-known $\mathrm{C}_{59} \mathrm{~N}^{\bullet}$ radical signal where charge is localised on the carbon next to the nitrogen atom and the extracted hyperfine coupling constant $A=10.1 \mathrm{MHz}$ is due to the coupling to ${ }^{14} \mathrm{~N}$. At the highest temperature reached in this particular experiment, $T=300{ }^{\circ} \mathrm{C}$, another set of weaker peaks that flank the main triplet of lines are also observed. They were observed before in the solution experiments and were tentatively assigned to the additional hyperfine splitting with ${ }^{13} \mathrm{C}$ nuclei in its natural abundance. Heating the sample above $300{ }^{\circ} \mathrm{C}$ causes a gradual loss of the EPR signal, probably due to the thermal instability of [10]CPP belts. The anisotropic rotation of $\mathrm{C}_{59} \mathrm{~N}$ radicals entrapped in [10]-cycloparaphenylene rings may not fully average out g-factor anisotropy, and hence the measured EPR spectrum measured at $290^{\circ} \mathrm{C}$ is broadened compared to the signal measured in pristine $\mathrm{C}_{59} \mathrm{~N}$ powders.

Fitting the EPR spectra to two overlapping components (i.e., the Lorentzian at $g=2.0022$ and the triplet of lines centered at $g=2.0014$ ) allows us to follow the evolution of signal intensities for these species (Figure 2) and compare their signal to $\mathrm{C}_{59} \mathrm{~N}^{\bullet}$ radicals acquired in the absence of [10]CPP (Figure 2b). The comparison shows some interesting differences which we attribute to the crucial presence of [10]CPP rings. Firstly, the $\mathrm{C}_{59} \mathrm{~N}^{\bullet} \mathrm{EPR}$ signal is observed for the first time at lower temperature in $\mathrm{C}_{59} \mathrm{~N}^{*} \subset[10] \mathrm{CPP}$ and is stronger as compared to unprotected azafullerenyl radicals by [10]CPP rings by about two orders of magnitude. The calibration of signal intensity shows that the highest concentration of $\mathrm{C}_{59} \mathrm{~N}^{\bullet} \subset[10] \mathrm{CPP}$ radicals is about 450 ppm reached at $300{ }^{\circ} \mathrm{C}$. Interestingly, thermogravimetric analysis (TGA) on the parent 
[10]CPP $\supset\left(\mathrm{C}_{59} \mathrm{~N}\right)_{2} \subset[10] \mathrm{CPP}$ complex demonstrated superior stability up to $\sim 300{ }^{\circ} \mathrm{C}$, as indicated by the very small weight loss (1.3\%), under ambient heating conditions up to this temperature (see Figure S7), while Raman spectroscopy verified the structural integrity of the complex (see Figure S2). Another surprising property of this signal is that it remains remarkably stable upon cooling as the signal intensity only marginally decreases with decreasing temperature. This points towards the high stability of $\mathrm{C}_{59} \mathrm{~N}^{\bullet}$ when protected by the [10]CPP rings. This observation is markedly different compared to bare $\mathrm{C}_{59} \mathrm{~N}^{\bullet}$ radicals in pristine $\left(\mathrm{C}_{59} \mathrm{~N}\right)_{2}$, where the re-dimerization kinetics is very fast and the signal intensity on cooling retraces that measured on warming (Figure $2 \mathrm{~b}$ ).
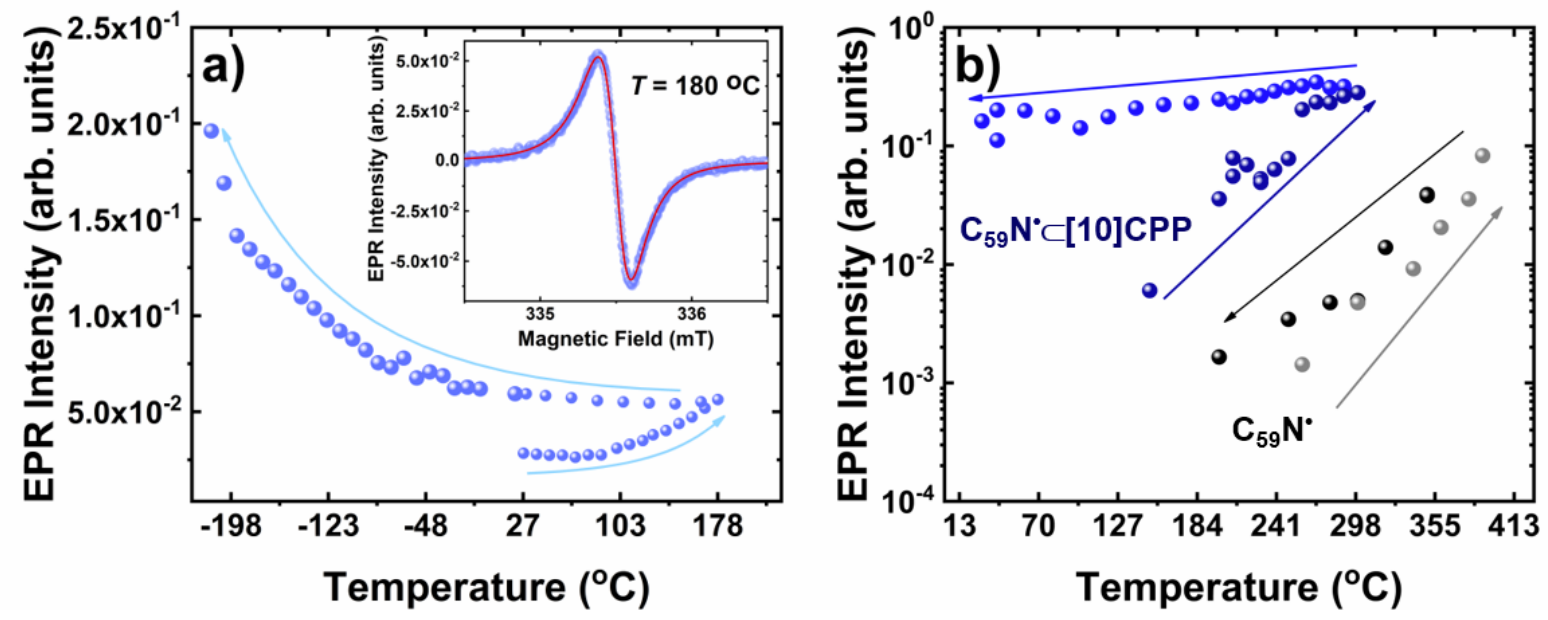

Figure 2. EPR signal intensities of $\mathrm{C}_{59} \mathrm{~N}$ species upon heating and cooling. (a) Temperature dependence of the X-band EPR signal intensity for the $g=2.0022$ spectra in a sample that initially already contained some of the $g=2.0022$ signal. In the inset we show a characteristic spectrum measured at $180^{\circ} \mathrm{C}$ together with a fit to the Lorentzian lineshape (thin red line). (b) Temperature dependences of normalised X-band EPR signal intensities for $\mathrm{C}_{59} \mathrm{~N} \bullet \mathrm{I}[10] \mathrm{CPP}$ radical signal (violet spheres are representing the heating and blue spheres the cooling) and compared to the $\mathrm{C}_{59} \mathrm{~N} \bullet$ measured for the pristine $\left(\mathrm{C}_{59} \mathrm{~N}\right)_{2}$ powder (grey spheres are representing the heating and black spheres the cooling). Arrows indicate temperature protocol used in these experiments.

The stability of $\mathrm{C}_{59} \mathrm{~N}^{*} \subset[10] \mathrm{CPP}$ radicals is next demonstrated by measuring the EPR signal at room temperatures as a function of time (Figure 3a): after $\sim 3$ days the signal marginally decreases and has comparable intensity even after 158 days. Quite remarkably, the signal can still be easily observed in the sample after 30 months (Figure $3 b$ and Figure S8), unlike bare $\mathrm{C}_{59} \mathrm{~N}^{\bullet}$ radicals in pristine $\left(\mathrm{C}_{59} \mathrm{~N}\right)_{2}$ powders, where no EPR signal can be detected at room 
temperature. Storage is under vacuum conditions, since exposure to air leads to significant degradation in signal strength over a timescale of minutes (Figure S5b).
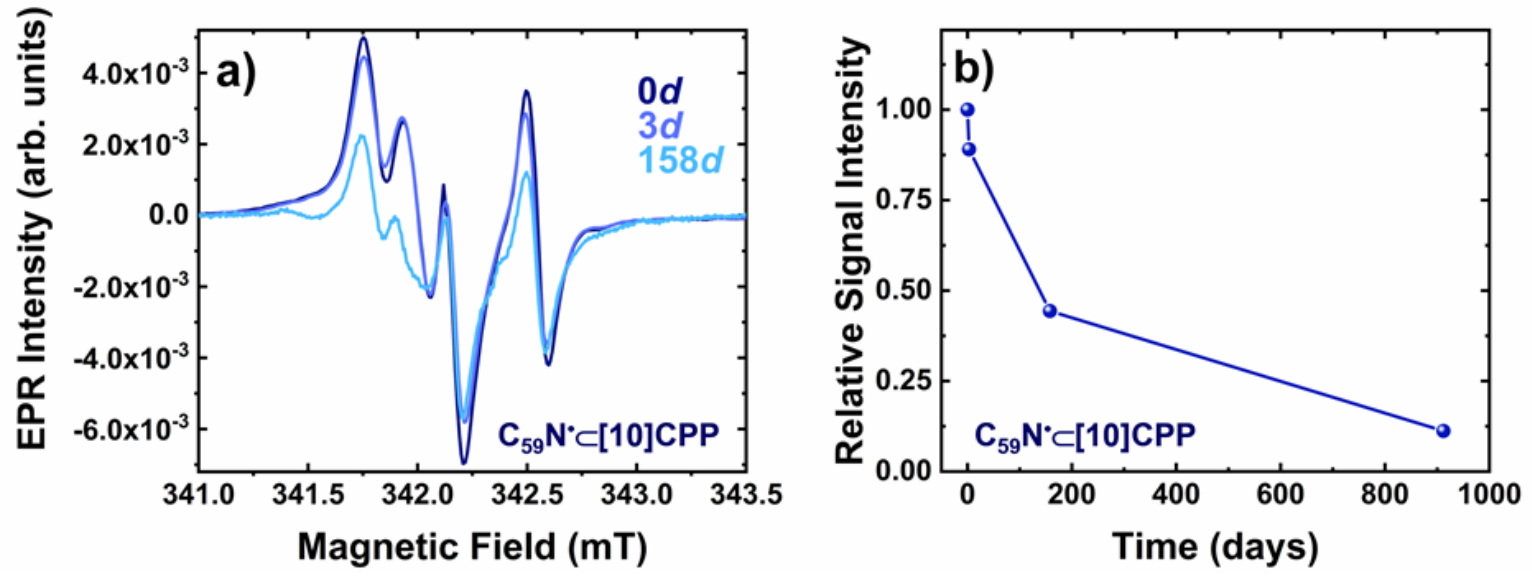

Figure 3. EPR signal intensities measured at room temperature. (a) The evolution of the room temperature $\mathrm{C}_{59} \mathrm{~N}^{*} \subset[10] \mathrm{CPP}$ radical signal over time span of 158 days and (b) the time dependence of the corresponding EPR signal relative intensity (normalised to 1 for $t=0$ ). The solid line in (b) is a guide to the eye.

The concentration of the $g=2.0022$ intermediate temperature spin species also shows large hysteresis in the X-band EPR signal intensity upon heating to $180^{\circ} \mathrm{C}$ and then cooling (Figure 2a, Figure S9), implying their high stability once they are formed. Cooling the sample well below room temperature reveals for the $g=2.0022$ signal a Curie-like dependence of the EPR signal intensity (Figure 2a), which is consistent with weak interactions between these radical centers. Since both $g=2.0014$ and $g=2.0022$ signals coexist at room temperature after hightemperature treatment, it is reasonable to suggest that thermal annealing indeed created two different and very stable radical states on $\mathrm{C}_{59} \mathrm{~N}$ cages when entrapped in [10]CPP.

EPR thus shows three distinct radical signatures: the first is due to a pre-existing signal present in the virgin sample at room temperature (previously assigned to $\mathrm{C}_{60}{ }^{-}$), the second appearing at intermediate temperatures as the first disappears, and the third, a major signal appearing at higher temperatures assigned to $\mathrm{C}_{59} \mathrm{~N}^{\bullet}$. We next explore the origins of these signals and attempt assignments using DFT calculations.

\subsection{Theoretical exploration and assignments of EPR signals by DFT calculations}

The absence of ${ }^{1} \mathrm{H}$ hyperfine coupling in experimental ESEEM measurements shows that hydrogenated species are not involved in the three EPR centers, and also excluded are reactions between $\mathrm{C}_{59} \mathrm{~N}^{\bullet}$ and [10]CPP (Figure S5, S6). This, therefore, leaves only azafullerenyl centers 
as the source for this signal (structures in Figure S10 and calculated hyperfine coupling parameters in Tables S1-1-4). The [10]CPP nanobelts are omitted from the DFT calculations for simplicity, since they do not contribute to the emerging EPR signal. They nonetheless have a critical impact on the persistence of the recorded signals upon annealing, as discussed below. Concerning the EPR signal witnessed at room temperature in the virgin sample we propose the presence of $\left(\mathrm{C}_{59} \mathrm{~N}\right)_{2}{ }^{-}$ions, by analogy to trace $\mathrm{C}_{60}{ }^{-}$ion EPR signal seen in as-prepared $\mathrm{C}_{60}$ samples. ${ }^{[22]}$ Unlike $\mathrm{C}_{59} \mathrm{~N}^{\bullet}$, in $\left(\mathrm{C}_{59} \mathrm{~N}\right)_{2}{ }^{-}$the unpaired electron is uniformly delocalised over both fullerene cages with almost no character on the nitrogen atoms (Figure S10c). This is very close in behaviour to the radical distribution of $\mathrm{C}_{60}{ }^{-}$(Figure S10a), but with higher and more uniform delocalisation. Furthermore, a calculation with both $\mathrm{C}_{60}$ and $\left(\mathrm{C}_{59} \mathrm{~N}\right)_{2}$ in the same supercell with an additional electron finds a ground state with the electron distributed primarily over the $\left(\mathrm{C}_{59} \mathrm{~N}\right)_{2}$, thus even if $\mathrm{C}_{60}{ }^{-}$was present there would be charge transfer to neighbouring azafullerene dimers. The distributed nature of the radical state and low hyperfine coupling parameters (see Table S1-1, S1-3) suggest that the EPR signal for $\mathrm{C}_{60}{ }^{-}$and $\left(\mathrm{C}_{59} \mathrm{~N}\right)_{2}{ }^{-}$should be largely indistinguishable spectroscopically. For these reasons we therefore reassign the $g=$ 1.9988 peak seen at room temperature in these and literature azafullerene samples to $\left(\mathrm{C}_{59} \mathrm{~N}\right)_{2}{ }^{-}$. The next radical signature is the signal observed at intermediate temperatures with $g=2.0022$. Herein, we exclude the triplet state for $\left(\mathrm{C}_{59} \mathrm{~N}\right)_{2}$, since we previously calculated this to be 0.87 $1.09 \mathrm{eV}$ less stable than the EPR silent $S=0$ ground state (the energy range depending on the presence or not of [10]CPP). ${ }^{21}$ At temperatures of about $100-150{ }^{\circ} \mathrm{C}$ it will simply not be possible to thermally populate such high-energy EPR active triplet state.

Creating the $g=2.0014 \mathrm{C}_{59} \mathrm{~N}^{\bullet}$ signal requires $1.80 \mathrm{eV}$ to completely break the intramolecular $\mathrm{C}-\mathrm{C}$ bond of $\left(\mathrm{C}_{59} \mathrm{~N}\right)_{2}\left(0.90 \mathrm{eV}\right.$ per $\mathrm{C}_{59} \mathrm{~N}^{\bullet}$ radical $)$. Given the $g=2.0022$ signal appears at lower temperatures, we would expect it to come from a more thermodynamically accessible species. We propose that the dimer bond does not fully dissociate, but instead breaks briefly and the resulting azafullerene cage rotates before recombining. This process can repeat multiple times, resulting in a metastable structure where the unpaired electron of one $\mathrm{C}_{59} \mathrm{~N}^{\bullet}$ binds to the back of the second $\mathrm{C}_{59} \mathrm{~N}^{*}$, analogous to $\mathrm{C}_{59} \mathrm{~N}-\mathrm{C}_{59} \mathrm{HN}$ back-bonded species seen during hightemperature treatment of $\mathrm{C}_{59} \mathrm{HN}$ powder. ${ }^{20}$ The extreme case where one cage has rotated through $180^{\circ}$ is $0.79 \mathrm{eV}$ less stable than the ground state $\left(\mathrm{C}_{59} \mathrm{~N}\right)_{2}\left(0.40 \mathrm{eV}\right.$ per $\left.\mathrm{C}_{59} \mathrm{~N}\right)$, significantly more stable than the formation of separated $\mathrm{C}_{59} \mathrm{~N}^{\bullet}$ radicals. Nudged elastic band calculations show the rate-limiting barrier for this rotation process is $1.50 \mathrm{eV}$ (Figure S11), below the calculated $1.80 \mathrm{eV}$ required to fully break the dimer bond. 
This rotated back-bonded $\mathrm{C}_{59} \mathrm{~N}-\mathrm{C}_{59} \mathrm{~N}$ dimer does not have unpaired spin in its ground state configuration (the triplet is $0.49 \mathrm{eV}$ less stable and so again not thermally accessible), and therefore is not a candidate for the EPR signal. However, the C-N at one end of the rotated $\mathrm{C}_{59} \mathrm{~N}-\mathrm{C}_{59} \mathrm{~N}$ dimer is now exposed and has the possibility to covalently bind to a neighbouring $\left(\mathrm{C}_{59} \mathrm{~N}\right)_{2}$ dimer (Figure 4). This is exothermic, releasing $0.19 \mathrm{eV}$ and forming an azafullerene tetramer that is stable in the triplet spin state $\left(\mu=2 \mu_{\mathrm{B}}\right)$ (Figure S10e). The two spins are very weakly coupled, with the anti-ferromagnetic configuration $13 \mathrm{meV}$ more stable than ferromagnetic pairing, and $36 \mathrm{meV}$ more stable than the paramagnetic solution.

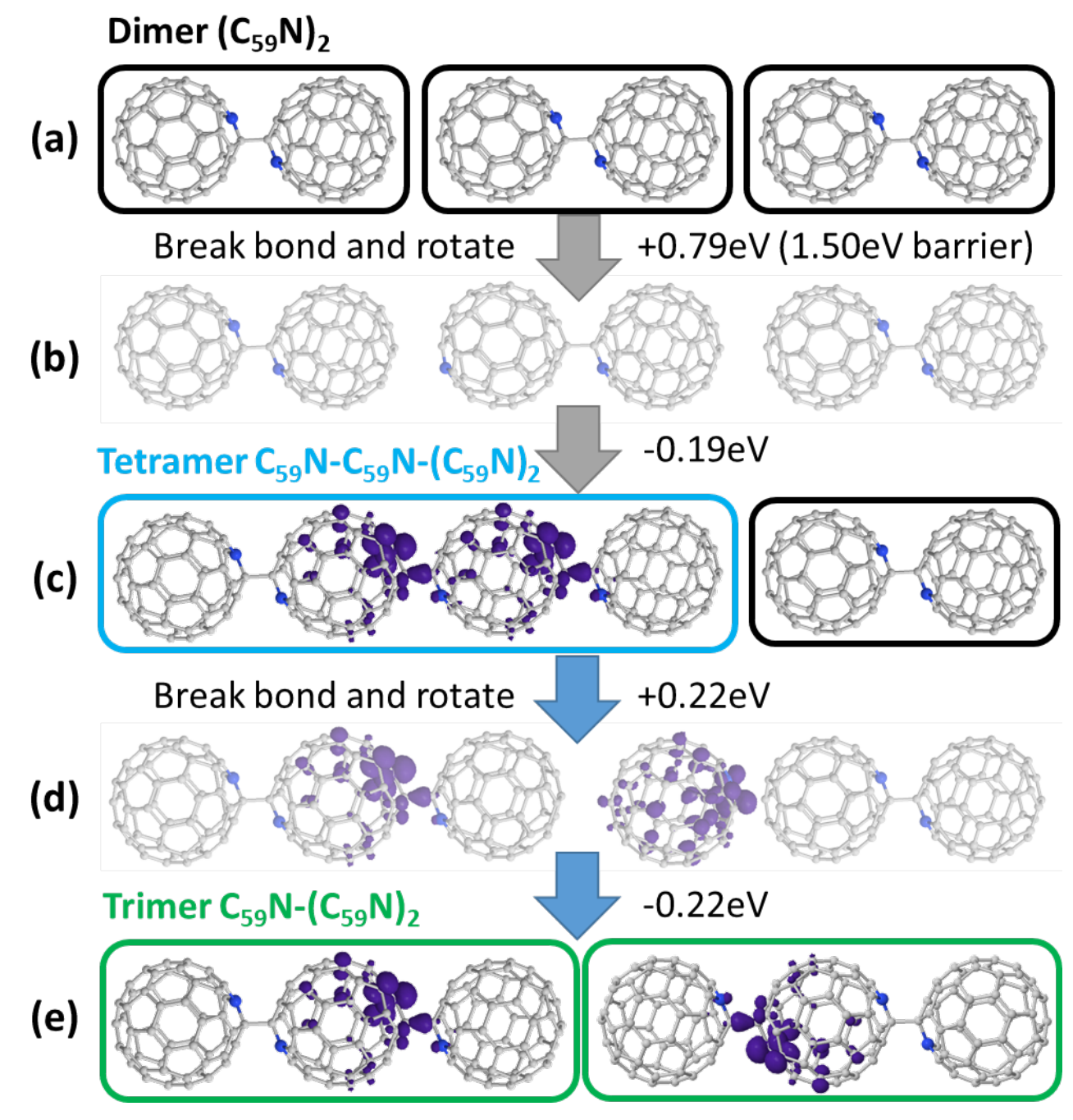

Figure 4. Schematic showing the bonding breaking and reformation processes going from linear chains of $\left(\mathrm{C}_{59} \mathrm{~N}\right)_{2}$ (black) to tetramers (blue) and trimers (green). Unpaired electron probability isosurfaces are shown (cutoff $0.002 \mathrm{e} / \AA^{3}$ ). From top to bottom the structures are (a) $3\left(\mathrm{C}_{59} \mathrm{~N}\right)_{2},(\mathrm{~b})\left(\mathrm{C}_{59} \mathrm{~N}\right)_{2}+\mathrm{C}_{59} \mathrm{~N}-\mathrm{C}_{59} \mathrm{~N}+\left(\mathrm{C}_{59} \mathrm{~N}\right)_{2},(\mathrm{c})\left(\mathrm{C}_{59} \mathrm{~N}\right)_{4} \cdot{ }^{\bullet}+\left(\mathrm{C}_{59} \mathrm{~N}\right)_{2},(\mathrm{~d})\left(\mathrm{C}_{59} \mathrm{~N}\right)_{3}{ }^{\bullet}+\mathrm{C}_{59} \mathrm{~N}^{\bullet}+$ $\left(\mathrm{C}_{59} \mathrm{~N}\right)_{2}(\mathrm{e}) 2\left(\mathrm{C}_{59} \mathrm{~N}\right)_{3}{ }^{\circ}$. 
Figure 4 shows this tetramer can easily lose an azafullerenyl radical, which can rotate and bond to a neighbouring dimer. The unpaired electron in each resulting trimer is localised on the back of the fullerene cages, far from any nitrogen atoms and hence will not show ${ }^{14} \mathrm{~N}$-induced hyperfine splitting (Figure 4e). It is nonetheless more localised than the spin state distribution in $\mathrm{C}_{60}{ }^{-}$and $\left(\mathrm{C}_{59} \mathrm{~N}\right)_{2}{ }^{-}$and so should show a distinct EPR signal (calculated hyperfine coupling coefficients are given in Table S1). We note that back-bonding is likely possible to multiple sites on the rear of the dimer, suggesting a family of related structures, each with slightly different weak hyperfine coupling parameters. Thermally activated jumping between these multiple sites may lead to the narrowing of the $g$-factor anisotropy ${ }^{23}$ and the lineshape that is well described already by a simple Lorentzian form. The trimer and tetramer therefore represent strong candidates for the intermediate $g=2.0022$ signal. The lack of characteristic hyperfine splitting in the EPR spectra is consistent with the low calculated hyperfine coupling constants on nitrogen, and the formation barrier is consistent with this species appearing at slightly lower temperatures than isolated $\mathrm{C}_{59} \mathrm{~N}^{\bullet}$. The radical state lies relatively deep in the gap and will trap charge from $\left(\mathrm{C}_{59} \mathrm{~N}\right)_{2}{ }^{-}$, consistent with the loss of the room temperature $g=1.9988$ signal as the $g=2.0022$ signal appears (Figure 1b). The relatively broad nature of this signal (especially when compared to sharp lines of $\mathrm{C}_{59} \mathrm{~N}^{\bullet}$ ) is consistent with a range of linear and non-linear trimers and tetramers depending on the local inter-fullerene bonding site on the back of the cage. Finally, the electron distribution and hyperfine coupling constants are very similar to that of $\mathrm{C}_{60}-\mathrm{C}_{59} \mathrm{~N}^{\bullet}$, consistent with the similarity between the $g=2.0022$ signal and that of $\mathrm{C}_{60}-\mathrm{C}_{59} \mathrm{~N}^{\bullet}{ }^{\cdot 23]}$ Interestingly, the structures show some similarity to azafullerene oligomers hypothesised to form when azafullerene dimers are incorporated into carbon nanotube cavities. ${ }^{24}$

The incorporation of encapsulating [10]CPPs into the calculations will likely affect the relative energies and barriers. Notably, prior studies show that the barrier for dimer homolysis is higher in the presence of [10]CPP due to the additional energetic cost of separating neighbouring nanorings. ${ }^{21}$ At the same time inter-ring interaction should serve to stabilise trimer and tetramer structures in the powder. The calculations also do not take into account any effects of $[10] \mathrm{CPP} \supset\left(\mathrm{C}_{59} \mathrm{~N}\right)_{2} \subset[10] \mathrm{CPP}$ packing in the crystal, which will be different with and without the presence of [10]CPP. These factors may explain why this intermediate signal is not observed in pure $\left(\mathrm{C}_{59} \mathrm{~N}\right)_{2}$ crystals without the presence of [10]CPP. A similar signal observed previously in the $\mathrm{C}_{59} \mathrm{~N}-\mathrm{C}_{60}$ complex ${ }^{23}$ disappears at around $90{ }^{\circ} \mathrm{C}$, a slightly lower temperature than in our case of [10]CPP $\supset\left(\mathrm{C}_{59} \mathrm{~N}\right)_{2} \subset[10] \mathrm{CPP}$ solid. This is yet further indirect evidence that the [10]CPP rings indeed stabilise the trimers. 
Moving to higher $\left(>150^{\circ} \mathrm{C}\right)$ annealing temperatures the primary EPR signal owing a $g=2.0014$ triplet is observed, attributed to the [10]CPP-enclosed $\mathrm{C}_{59} \mathrm{~N}^{\bullet}$. The formation of $\mathrm{C}_{59} \mathrm{~N}^{\bullet}$ from trimers has an enthalpic cost, increased still further by the energy to separate neighboring [10]CPPs. However, this is offset by entropy gains associated with transformation from the low entropy configuration of the azafullerene trimer chain held within interacting nanorings. Separation into $\mathrm{C}_{59} \mathrm{~N}^{*} \subset[10] \mathrm{CPP}$ frees up molecular rotation of all species, and allows the possibility of local structural reorganization of the crystal. $\mathrm{C}_{60} \subset[10] \mathrm{CPP}$ is known to form a herringbone type structure, the $\mathrm{C}_{60}$ molecules spatially separated from one another by the nanorings ${ }^{25}$ and similar structural motifs are expected also in the $\mathrm{C}_{59} \mathrm{~N}^{*} \subset[10] \mathrm{CPP}$ case.

\subsection{Encapsulated azafullerene radical $\mathrm{C}_{59} \mathrm{~N}^{\bullet} \subset[10] \mathrm{CPP}$ as a robust spin-coherent quantum unit}

Figure S10b shows the unpaired electron in $\mathrm{C}_{59} \mathrm{~N}^{\bullet}$ is highly localised on the carbon neighbouring the nitrogen atom, with a calculated ${ }^{14} \mathrm{~N}$ hyperfine coupling constant $A$ of 10.76 $\mathrm{MHz}$, in excellent agreement with our experimental value of $10.1 \mathrm{MHz}$. It is somewhat intriguing that the [10]CPP has little effect on the ${ }^{14} \mathrm{~N}$ hyperfine coupling constant as it is nearly the same as in bare $\mathrm{C}_{59} \mathrm{~N}^{\bullet}$ cages. ${ }^{16-18,20}$ This is very different to the case of $\mathrm{N} @ \mathrm{C}_{60}$ where confinement inside the fullerene molecules increases the $\mathrm{N}$ hyperfine coupling $(A=15.73 \mathrm{MHz})$ by $\sim 50 \%$ compared to that of atomic nitrogen. ${ }^{11}$

While this signal is itself well known and understood, the unambiguously fascinating experimental finding of this work is its long-term stability in the solid when entrapped in [10]CPP rings. Such high stability together with the possibilities offered by [10]CPP chemistry to custom rationally designed $\mathrm{C}_{59} \mathrm{~N}$ spin networks may find its application in quantum computation. For such applications, the coherence times need to be sufficiently long. They were measured for $\mathrm{C}_{59} \mathrm{~N}^{*} \subset[10] \mathrm{CPP}$ on a powder sample previously heated to $290{ }^{\circ} \mathrm{C}$ using pulsed EPR methods. The Fourier transform of the echo signal indeed shows the dominant $\mathrm{C}_{59} \mathrm{~N}^{\bullet}$ coexisting with the weaker $g=2.0022$ trimer/tetramer component (Figure S12a,c). Next, the memory time $T_{\mathrm{M}}=631 \mathrm{~ns}$ and the spin-lattice relaxation time $T_{1}=210 \mu \mathrm{s}$ (the two-exponential magnetization recovery is probably due to the presence of $g=2.0022$ component with $T_{1}=8.1$ $\mu \mathrm{s})$ were measured at room temperature using a standard echo-decay and inversion-recovery pulse sequences (Figure S12b,d). On cooling, relaxation times rapidly increase and $T_{1}$ is already in the range of ms below $200 \mathrm{~K}$ (Figure S13). 


\section{Discussion and Conclusion}

These room temperature coherence times for $\mathrm{C}_{59} \mathrm{~N}^{\bullet} \subset[10] \mathrm{CPP}$ are comparable to relaxation times measured at $-83^{\circ} \mathrm{C}$ for $\mathrm{N} @ \mathrm{C}_{60}$ in powder $\left(T_{\mathrm{M}}=5 \mu \mathrm{s}\right.$ and $\left.T_{1}=120 \mu \mathrm{s}\right)$ in peapods $\left(T_{\mathrm{M}}=\right.$ $\left.T_{1}=13 \mu \mathrm{s}\right),{ }^{26}$ and at $5 \mathrm{~K}$ in graphenoids $\left(T_{M}=300 \mathrm{~ns}, T_{1}=100 \mu \mathrm{s}\right)^{27}$ which supports [10]CPP $\supset\left(\mathrm{C}_{59} \mathrm{~N}\right)_{2} \subset[10] \mathrm{CPP}$ as a promising candidate to build qubit circuits. Temperature dependent measurements show that $T_{1}$ rapidly increases as the system is cooled (Figure S13). At $-83^{\circ} \mathrm{C} T_{1}$ is already 2.89(6) ms, over 20 times longer than that of $\mathrm{N} @ \mathrm{C}_{60}$. On further cooling it becomes so long that it can no longer be reliably determined with our pulsed EPR spectrometer, approaching the range of seconds.

The ability to prepare the initial electronic spin state in the arbitrary superposition of electron $m_{S}$ levels is here demonstrated by the observation of many Rabi oscillations extending over several $\mu$ s time scale (Figure S14). We note one more time, that $\mathrm{C}_{59} \mathrm{~N}^{*} \subset[10] \mathrm{CPP}$ signal also in this aspect show very similar performance as highly diluted and expensive N@ $\mathrm{C}_{60}$ in frozen $\mathrm{CS}_{2}{ }^{28}$ These experiments thus establish $\mathrm{C}_{59} \mathrm{~N}^{\bullet} \subset[10] \mathrm{CPP}$ centers as a platform where coherent manipulation of the electron spin state (qubit) with a resonant microwave pulse can easily be achieved.

We note that the concept of entrapping spin-active fullerene molecules may not be limited just to the $\mathrm{C}_{59} \mathrm{~N}$ case presented in this study. For example, recent studies of $\mathrm{Ln}_{2} @ \mathrm{C}_{79} \mathrm{~N}^{29}$ demonstrated enormous ferromagnetic exchange between Ln and a azafullerene radical. When such single molecule magnets (SMM) are hosted by [10]-cycloparaphenylene rings, the interaction between individual SMM moments can be tailored thus enabling bottom-up design of SMM lattices and tuning of the interactions between SMM entities.

It has previously been proposed that dipolar interactions between neighboring fullerenes could be used to control their coupling in qubit networks. ${ }^{30}$ In our current case, we estimate this interqubit coupling to be $\sim 10 \mathrm{MHz}$ (see Supplementary Text), and this could be locally controlled by designing inter-fullerene spacing and $\mathrm{C}_{59} \mathrm{~N}^{\bullet}$ orientation via the host [10]CPP chemistry.

Furthermore, careful temperature control of the annealing should allow tuning of the relative ratio of $\mathrm{C}_{59} \mathrm{~N}^{\bullet}$ to trimer/tetramer spin species. While the $\mathrm{C}_{59} \mathrm{~N}^{\bullet} \subset[10] \mathrm{CPP}$ spin species could clearly play the role of a qubit, the other trimer/tetramer spin species could be explored for coupling between individual qubits. Manipulation of these species by an external bias (microwave heating and/or light irradiation) may pave the wave towards high output functional devices. Additional design flexibility is offered both by recent advances in the synthesis of CPPs as well as developed upscale production methods. Combined with the ease of access to 
macroscopic amounts of the parent diamagnetic $\left(\mathrm{C}_{59} \mathrm{~N}\right)_{2}$ via conventional chemistry routes, these constitute key factors towards realization of extended circuits based on [10]CPP nanorings and spin-active azafullerene cages. Collectively, the addressed properties seem to challenge the long-standing materials problems of molecular spin entities as candidates for the quantum circuits of qubits.

\section{Materials and Methods}

Preparation of the $[10] C P P \neg\left(C_{59} N\right)_{2} \subset[10] C P P$ powder samples

In previous studies on the complexation of $\left(\mathrm{C}_{59} \mathrm{~N}\right)_{2}$ and [10]CPP towards the formation of the [10]CPP $\supset\left(\mathrm{C}_{59} \mathrm{~N}\right)_{2} \subset[10] \mathrm{CPP}$ complex in 1-chloronaphthalene, we described the cooperative complexation mechanism furnishing the desired 2:1 complex in solution. ${ }^{31}$ In this current study, we have prepared the [10]CPP $\supset\left(\mathrm{C}_{59} \mathrm{~N}\right)_{2} \subset[10] \mathrm{CPP}$ complex by mixing the individual components in a 2:1 molar ratio in 1-chloronaphthalene. $8.0 \mathrm{mg}(1.0 \mathrm{eq}, 5.5 \mu \mathrm{mol})$ of $\left(\mathrm{C}_{59} \mathrm{~N}\right)_{2}$ were dissolved in $0.9 \mathrm{~mL}$ 1-chloronaphthalene, while $8.4 \mathrm{mg}$ [10]CPP (2.0 eq, $11 \mu \mathrm{mol})$ were dissolved in $1.7 \mathrm{~mL}$ 1-chloronaphthalene. Then, the two solutions were mixed in a $4 \mathrm{~mL}$ glass vial and stirred at rt for $3 \mathrm{~d}$. After that period the mixture was let to settle overnight in a refrigerator. Then a layer of cold hexane $(1 \mathrm{~mL})$ was carefully placed on top of the 1chloronaphthalene layer and refrigerated for another 24h. Finally, the solid residual was mildly centrifuged (5 min, $1600 \mathrm{rpm}$ ), washed once with cold hexane and dried under vacuum. Comparison of solid state ${ }^{1} \mathrm{H}$ MAS and ${ }^{13} \mathrm{C}$ CP MAS NMR spectra measured on reference [10]CPP and $\left(\mathrm{C}_{59} \mathrm{~N}\right)_{2}$ powders with our product [10]CPP $\supset\left(\mathrm{C}_{59} \mathrm{~N}\right)_{2} \subset[10] \mathrm{CPP}$ powders clearly shows shifts of peaks in the latter case, which unambiguously confirm the interaction between the azafullerene and [10]CPP (see Figure S3 and associated discussion). Thermogravimetric analysis, FT-IR and Raman spectroscopy data for the samples are given Supplementary in Figure S2, S7 and associated discussion.

\section{Nuclear magnetic resonance}

Measurements were performed with a Bruker Advance III $500 \mathrm{MHz}$ high-resolution solid-state NMR spectrometer equipped with $11.74 \mathrm{~T}$ Bruker Ultrashield superconducting magnet. Powders were packed into rotors with a diameter of $2.5 \mathrm{~mm}$ and two tiny Teflon inserts for better filling and rotation. Magic angle spinning experiments at rotation frequencies of $35 \mathrm{kHz}$ and $15 \mathrm{kHz}$ were employed in ${ }^{1} \mathrm{H}$ and ${ }^{13} \mathrm{C}$ NMR measurements, respectively. The ${ }^{1} \mathrm{H}$ and ${ }^{13} \mathrm{C}$ Larmor frequencies were $500 \mathrm{MHz}$ and $125.8 \mathrm{MHz}$ and the chemical shift referencing is reported against the adamantane reference. For the ${ }^{1} \mathrm{H}$ MAS NMR experiments 32 signals were 
accumulated using echo pulse sequence with a $\pi / 2$ pulse-length of $2.5 \mu$ s, interpulse delay of $10 \mu$ s and repetition time of $1 \mathrm{~s}$. For the ${ }^{13} \mathrm{C}$ MAS NMR experiments 4096 signals were accumulated using echo pulse sequence with a $\pi / 2$ pulse-length of $2.5 \mu$ s, interpulse delay of $10 \mu$ s and repetition time of $8 \mathrm{~s}$. All experiments were performed at room temperature.

\section{Thermogravimetric Analysis}

TGA analysis was performed via a TA Q500 instrument operated by a personal computer. The samples were analysed at nitrogen atmosphere with a temperature ramp of $10^{\circ} \mathrm{C} / \mathrm{min}$.

\section{Fourier-Transform Infra-Red Spectroscopy and Raman spectroscopy}

FT IR spectra were recorded on Bruker Equinox 55 FTIR spectrometer equipped with a Pike Miracle Ge ATR accessory at room temperature. Micro-Raman scattering measurements were performed at room temperature in the backscattering geometry using a RENISHAW inVia Raman microscope equipped with a CCD camera and a Leica microscope. A He/Nelaser (633 $\mathrm{nm})$ and 2400 lines/mm grating were used, providing a spectral resolution of $\pm 1 \mathrm{~cm}^{-1}$. Measurements were taken with 10 s seconds of exposure times and laser power $\sim 0.3 \mathrm{~mW} / \mathrm{cm}^{2}$ to prevent overheating. The laser spot was focused on the sample surface using a long working distance 50x (L50) objective.

\section{Electron paramagnetic resonance}

Around 5-10 mg of [10]CPP $\supset\left(\mathrm{C}_{59} \mathrm{~N}\right)_{2} \subset[10] \mathrm{CPP}$ powder was sealed under the dynamic vacuum in a $4 \mathrm{~mm}$ diameter silica tube (Wilmad Lab Glass). High-temperature experiments were performed on a home-built X-band EPR spectrometer equipped with a Varian E-101 microwave bridge and a Varian TEM104 dual cavity resonator. The high-temperature unit allows for continuous-wave (cw) EPR experiments up to $350^{\circ} \mathrm{C}$ without compromising the quality of the resonator. The temperature stability is better than $\pm 2 \mathrm{~K}$. Pulsed X-band EPR experiments were performed on the same samples but on the Bruker E580 spectrometer. Here, the field-swept spectra were recorded by measuring the Hahn-echo intensity (the pulse sequence $\tau_{\mathrm{p}}(\pi / 2)-\tau-$ $\tau_{\mathrm{p}}(\pi)-\tau$-echo with $\tau_{\mathrm{p}}(\pi / 2)=16 \mathrm{~ns}$ and $\left.\tau=200 \mathrm{~ns}\right)$. For the spin-spin memory time $\left(T_{\mathrm{M}}\right)$ and ESEEM experiments interpulse delay time $\tau$ of the Hahn-echo pulse sequence systematically increased from its initial value of $200 \mathrm{~ns}$. The spin-lattice relaxation time was measured using inversion-recovery technique and a Hahn-echo readout, $\tau_{\mathrm{p}}(\pi)-T-\tau_{\mathrm{p}}(\pi / 2)-\tau-\tau_{\mathrm{p}}(\pi)-\tau$-echo. For the measurements of the Rabi oscillations, we used a nutation experiment with the echo detection. A nutation microwave pulse that coherently drives the electron magnetization was 
varied from the initial pulse length of 16 ns to the maximum of 2000 ns. The microwave field $B_{1}$ was varied by using different microwave attenuations and calibrated by measuring the pulse length of $\pi / 2$ pulse. In all cases appropriate phase cycling was applied to compensate for the pulse errors.

\section{Density Functional Theory (DFT) Calculations}

DFT calculations were performed using the AIMPRO code ${ }^{32-34}$ under the local density approximation (LDA). The spin-polarised and -averaged charge density was fitted to plane waves with energy cut-off of $150 \mathrm{Ha}$ for $\mathrm{C}$ and $175 \mathrm{Ha}$ for N, while Kohn-Sham wave functions were constructed using localized Cartesian Gaussian orbital functions (38 for C, 40 for N, $l \leq$ 2). Relativistic pseudopotentials were used, ${ }^{35}$ with a finite electron Fermi temperature of $\mathrm{kT}=0.04 \mathrm{eV}$. Large hexagonal supercells $\left(26.46 \times 26.46 \AA\right.$ for $\mathrm{C}_{59} \mathrm{~N}$ and $\mathrm{C}_{60}, 26.46 \times 37.04 \AA$ for $2 \mathrm{C}_{59} \mathrm{~N}, 26.46 \times 47.63 \AA$ for $3 \mathrm{C}_{59} \mathrm{~N}, 26.46 \times 58.21 \AA$ for $4 \mathrm{C}_{59} \mathrm{~N}$, and $46.46 \times 79.38 \AA$ for $6 \mathrm{C}_{59} \mathrm{~N}$ ) ensured $>20 \AA$ vacuum between neighboring systems, with a single k-point. All atomic positions were independently optimized to convergence in position of $10^{-5} \mathrm{Ha}$ and energy $10^{-7} \mathrm{Ha}$. Energy differences in Figure 4 are calculated from cells featuring six independent $\mathrm{C}_{59} \mathrm{~N}$. Hyperfine coupling parameters are calculated after all-electron wavefunction core reconstruction, ${ }^{36}$ with both LDA and generalized gradient approximation tested for comparison (see Table S1).

\section{Author Contributions:}

Yuri Tanuma, Jeremy Rio and Chris Ewels performed DFT calculations, Anastasios Stergiou, Nikos Tagmatarchis, Jannis Volkmann, and Hermann A. Wegner synthesized and characterized azafullerene species and cycloparaphenylenes, Andreja Bužan Bobnar and Denis Arčon performed EPR experiments, Mattia Gaboardi performed powder XRD. The work was conceived and developed by Hermann Wegner, Nikos Tagmatarchis, Chris Ewels and Denis Arčon. All authors contributed to analysis and interpretation of the data, and production and editing of the manuscript.

Conflict of Interest: The authors have no financial or commercial Conflict of Interest to report.

\section{Acknowledgements}

Yuri Tanuma and Anastasios Stergiou contributed equally to this work. Authors would like to thank Alan Gregorovič (Institute Jožef Stefan, Ljubljana, Slovenia) for assistance with NMR experiments. Authors acknowledge help and fruitful discussions with L. Petaccia and J. Plaisier (Sincrotrone Trieste, Trieste, Italy) on the powder XRD results. Y.T. acknowledges funding from French Government Scholarships and from Inoue Enryo Memorial Grant Toyo University. D.A. acknowledges financial support from the Slovenian Research Agency (Core Research Funding No. P1-0125 and Projects No. J1-9145 and No. N1-0052). Y.T., J. R. and C.E. thank the CCIPL (Center de Calculs Intensif Pays de la Loire) where DFT calculations were performed. C.E., J.R., Y.T., N.T. and H.W. acknowledge the Pays de la Loire Pari Scientifique project "NEWTUBE" for funding. D.A. and C.E. acknowledge BI-FR/21-22-PROTEUS-003 project for funding. Support of this work by the project "National Infrastructure in 
Nanotechnology, Advanced Materials and Micro-/Nanoelectronics” (MIS 5002772), implemented under the "Reinforcement of the Research and Innovation Infrastructures", funded by the Operational Program "Competitiveness, Entrepreneurship, and Innovation" (NSRF 2014-2020) and co-financed by Greece and the European Union (European Regional Development Fund), is acknowledged. Partial support by the programme for the promotion of the exchange and scientific cooperation between Greece and Germany, IKYDA 2020, is acknowledged.

\section{References}

(1) Gaita-Ariño, A.; Luis, F.; Hill, S.; Coronado, E. Molecular Spins for Quantum Computation. Nature Chemistry 2019, 11 (4), 301-309. https://doi.org/10.1038/s41557019-0232-y.

(2) Clemente-Juan, J. M.; Coronado, E.; Gaita-Ariño, A. Magnetic Polyoxometalates: From Molecular Magnetism to Molecular Spintronics and Quantum Computing. Chem. Soc. Rev. 2012, 41 (22), 7464. https://doi.org/10.1039/c2cs35205b.

(3) Aromí, G.; Aguilà, D.; Gamez, P.; Luis, F.; Roubeau, O. Design of Magnetic Coordination Complexes for Quantum Computing. Chem. Soc. Rev. 2012, 41 (2), 537 546. https://doi.org/10.1039/C1CS15115K.

(4) Sessoli, R. Toward the Quantum Computer: Magnetic Molecules Back in the Race. ACS Cent. Sci. 2015, 1 (9), 473-474. https://doi.org/10.1021/acscentsci.5b00384.

(5) Takabayashi, Y.; Ganin, A. Y.; Jeglič, P.; Arčon, D.; Takano, T.; Iwasa, Y.; Ohishi, Y.; Takata, M.; Takeshita, N.; Prassides, K.; Rosseinsky, M. J. The Disorder-Free NonBCS Superconductor Cs3C60 Emerges from an Antiferromagnetic Insulator Parent State. Science 2009, 323 (5921), 1585-1590. https://doi.org/10.1126/science.1169163.

(6) Ganin, A. Y.; Takabayashi, Y.; Jeglič, P.; Arčon, D.; Potočnik, A.; Baker, P. J.; Ohishi, Y.; McDonald, M. T.; Tzirakis, M. D.; McLennan, A.; Darling, G. R.; Takata, M.; Rosseinsky, M. J.; Prassides, K. Polymorphism Control of Superconductivity and Magnetism in Cs 3 C 60 Close to the Mott Transition. Nature 2010, 466 (7303), 221225. https://doi.org/10.1038/nature09120.

(7) Lappas, A.; Prassides, K.; Vavekis, K.; Arcon, D.; Blinc, R.; Cevc, P.; Amato, A.; Feyerherm, R.; Gygax, F. N.; Schenck, A. Spontaneous Magnetic Ordering in the Fullerene Charge-Transfer Salt (TDAE)C60. Science 1995, 267 (5205), 1799-1802. https://doi.org/10.1126/science.267.5205.1799.

(8) Benjamin, S. C.; Ardavan, A.; Briggs, G. A. D.; Britz, D. A.; Gunlycke, D.; Jefferson, J.; Jones, M. A. G.; Leigh, D. F.; Lovett, B. W.; Khlobystov, A. N.; Lyon, S. A.; Morton, J. J. L.; Porfyrakis, K.; Sambrook, M. R.; Tyryshkin, A. M. Towards a Fullerene-Based Quantum Computer. J. Phys.: Condens. Matter 2006, 18 (21), S867S883. https://doi.org/10.1088/0953-8984/18/21/S12.

(9) Morton, J. J. L.; Tyryshkin, A. M.; Ardavan, A.; Benjamin, S. C.; Porfyrakis, K.; Lyon, S. A.; Briggs, G. A. D. Bang-Bang Control of Fullerene Qubits Using Ultrafast Phase Gates. Nature Physics 2006, 2 (1), 40-43. https://doi.org/10.1038/nphys192.

(10) Morton, J. J. L.; Bertet, P. Storing Quantum Information in Spins and High-Sensitivity ESR. Journal of Magnetic Resonance 2018, 287, 128-139. https://doi.org/10.1016/j.jmr.2017.11.015.

(11) Almeida Murphy, T.; Pawlik, Th.; Weidinger, A.; Höhne, M.; Alcala, R.; Spaeth, J.-M. Observation of Atomlike Nitrogen in Nitrogen-Implanted Solid \$\{\mathrm\{C\}\}_\{60\}\$. Phys. Rev. Lett. 1996, 77 (6), 1075-1078. https://doi.org/10.1103/PhysRevLett.77.1075. 
(12) Morton, J. J. L.; Tyryshkin, A. M.; Ardavan, A.; Porfyrakis, K.; Lyon, S. A.; Briggs, G. A. D. A New Mechanism for Electron Spin Echo Envelope Modulation. J. Chem. Phys. 2005, 122 (17), 174504. https://doi.org/10.1063/1.1888585.

(13) Harneit, W.; Huebener, K.; Naydenov, B.; Schaefer, S.; Scheloske, M. N@C60 Quantum Bit Engineering. physica status solidi (b) 2007, 244 (11), 3879-3884. https://doi.org/10.1002/pssb.200776193.

(14) Waiblinger, M.; Lips, K.; Harneit, W.; Weidinger, A.; Dietel, E.; Hirsch, A. Corrected Article: Thermal Stability of the Endohedral Fullerenes \$ $\{\backslash \text { mathrm }\{\mathrm{N}\} \backslash \text { mathrm }\{@\} \backslash \operatorname{mathrm}\{\mathrm{C}\}\}_{-}\{60\}, \$ \$\{$ mathrm $\{\mathrm{N}\} \backslash$ mathrm $\{@\} \backslash$ mathrm $\{\mathrm{C}\}\}_{-}\{70\}$,\$ and $\$\{\backslash \text { mathrm }\{\mathrm{P}\} \backslash \text { mathrm }\{@\} \backslash \text { mathrm }\{\mathrm{C}\}\}_{-}\{60\}$ [Phys. Rev. B 63, 045421 (2001)]. Phys. Rev. B 2001, 64 (15), 159901. https://doi.org/10.1103/PhysRevB.64.159901.

(15) Hummelen, J. C.; Knight, B.; Pavlovich, J.; González, R.; Wudl, F. Isolation of the Heterofullerene C59N as Its Dimer (C59N)2. Science 1995, 269 (5230), 1554-1556. https://doi.org/10.1126/science.269.5230.1554.

(16) Gruss, A.; Dinse, K.-P.; Hirsch, A.; Nuber, B.; Reuther, U. Photolysis of (C59N)2 Studied by Time-Resolved EPR. J. Am. Chem. Soc. 1997, 119 (37), 8728-8729. https://doi.org/10.1021/ja971669h.

(17) Hasharoni, K.; Bellavia-Lund, C.; Keshavarz-K, M.; Srdanov, G.; Wudl, F. LightInduced ESR Studies of the Heterofullerene Dimers. J. Am. Chem. Soc. 1997, 119 (45), 11128-11129. https://doi.org/10.1021/ja972002y.

(18) Simon, F.; Arčon, D.; Tagmatarchis, N.; Garaj, S.; Forro, L.; Prassides, K. ESR Signal in Azafullerene (C59N)2 Induced by Thermal Homolysis. J. Phys. Chem. A 1999, 103 (35), 6969-6971. https://doi.org/10.1021/jp9912915.

(19) Keshavarz-K, M.; González, R.; Hicks, R. G.; Srdanov, G.; Srdanov, V. I.; Collins, T. G.; Hummelen, J. C.; Bellavia-Lund, C.; Pavlovich, J.; Wudl, F.; Holczer, K. Synthesis of Hydroazafullerene C 59 HN, the Parent Hydroheterofullerene. Nature 1996, 383 (6596), 147-150. https://doi.org/10.1038/383147a0.

(20) Arčon, D.; Pregelj, M.; Cevc, P.; Rotas, G.; Pagona, G.; Tagmatarchis, N.; Ewels, C. Stability, Thermal Homolysis and Intermediate Phases of Solid Hydroazafullerene C59HN. Chem. Commun. 2007, No. 32, 3386-3388. https://doi.org/10.1039/B703766J.

(21) Stergiou, A.; Rio, J.; Griwatz, J. H.; Arčon, D.; Wegner, H. A.; Ewels, C. P.; Tagmatarchis, N. A Long-Lived Azafullerenyl Radical Stabilized by Supramolecular Shielding with a [10]Cycloparaphenylene. Angewandte Chemie International Edition 2019, 58 (49), 17745-17750. https://doi.org/10.1002/anie.201909126.

(22) Science of Fullerenes and Carbon Nanotubes; Elsevier, 1996. https://doi.org/10.1016/B978-0-12-221820-0.X5000-X.

(23) Rockenbauer, A.; Csányi, G.; Fülöp, F.; Garaj, S.; Korecz, L.; Lukács, R.; Simon, F.; Forró, L.; Pekker, S.; Jánossy, A. Electron Delocalization and Dimerization in Solid \$ $\{\text { mathrm }\{\mathrm{C}\}\}_{-}\{59\} \backslash$ mathrm $\{\mathrm{N}\}$ \$ Doped $\$\{\backslash \text { mathrm }\{\mathrm{C}\}\}_{-}\{60\}$ S Fullerene. Phys. Rev. Lett. 2005, 94 (6), 066603. https://doi.org/10.1103/PhysRevLett.94.066603.

(24) Pagona, G.; Rotas, G.; Khlobystov, A. N.; Chamberlain, T. W.; Porfyrakis, K.; Tagmatarchis, N. Azafullerenes Encapsulated within Single-Walled Carbon Nanotubes. J. Am. Chem. Soc. 2008, 130 (19), 6062-6063. https://doi.org/10.1021/ja800760w.

(25) Xia, J.; Bacon, J. W.; Jasti, R. Gram-Scale Synthesis and Crystal Structures of [8]- and [10]CPP, and the Solid-State Structure of C60@[10]CPP. Chem. Sci. 2012, 3 (10), 3018-3021. https://doi.org/10.1039/C2SC20719B.

(26) Tóth, S.; Quintavalle, D.; Náfrádi, B.; Korecz, L.; Forró, L.; Simon, F. Enhanced Thermal Stability and Spin-Lattice Relaxation Rate of N@ C 60 inside Carbon Nanotubes. Phys. Rev. B 2008, 77 (21), 214409. https://doi.org/10.1103/PhysRevB.77.214409. 
(27) Lombardi, F.; Lodi, A.; Ma, J.; Liu, J.; Slota, M.; Narita, A.; Myers, W. K.; Müllen, K.; Feng, X.; Bogani, L. Quantum Units from the Topological Engineering of Molecular Graphenoids. Science 2019, 366 (6469), 1107-1110. https://doi.org/10.1126/science.aay7203.

(28) Morton, J. J. L.; Tyryshkin, A. M.; Ardavan, A.; Porfyrakis, K.; Lyon, S. A.; Briggs, G. A. D. Measuring Errors in Single-Qubit Rotations by Pulsed Electron Paramagnetic Resonance. Phys. Rev. A 2005, 71 (1), 012332. https://doi.org/10.1103/PhysRevA.71.012332.

(29) Dey, S.; Rajaraman, G. Attaining Record-High Magnetic Exchange, Magnetic Anisotropy and Blocking Barriers in Dilanthanofullerenes. Chemical Science 2021. https://doi.org/10.1039/D1SC03925C.

(30) Harneit, W. Fullerene-Based Electron-Spin Quantum Computer. Phys. Rev. A 2002, 65 (3), 032322. https://doi.org/10.1103/PhysRevA.65.032322.

(31) Rio, J.; Beeck, S.; Rotas, G.; Ahles, S.; Jacquemin, D.; Tagmatarchis, N.; Ewels, C.; Wegner, H. A. Electronic Communication between Two [10]Cycloparaphenylenes and Bis(Azafullerene) (C59 N)2 Induced by Cooperative Complexation. Angew Chem Int Ed Engl 2018, 57 (23), 6930-6934. https://doi.org/10.1002/anie.201713197.

(32) Briddon, P. R.; Jones, R. LDA Calculations Using a Basis of Gaussian Orbitals. physica status solidi (b) 2000, 217 (1), 131-171. https://doi.org/10.1002/(SICI)15213951(200001)217:1<131::AID-PSSB131>3.0.CO;2-M.

(33) Rayson, M. J. Rapid Filtration Algorithm to Construct a Minimal Basis on the Fly from a Primitive Gaussian Basis. Computer Physics Communications 2010.

https://doi.org/10.1016/j.cpc.2010.02.012.

(34) Briddon, P. R.; Rayson, M. J. Accurate Kohn-Sham DFT with the Speed of Tight Binding: Current Techniques and Future Directions in Materials Modelling. physica status solidi (b) 2011, 248 (6), 1309-1318. https://doi.org/10.1002/pssb.201046147.

(35) Hartwigsen, C.; Goedecker, S.; Hutter, J. Relativistic Separable Dual-Space Gaussian Pseudopotentials from H to Rn. Phys. Rev. B 1998, 58 (7), 3641-3662. https://doi.org/10.1103/PhysRevB.58.3641.

(36) Shaw, M. J.; Briddon, P. R.; Goss, J. P.; Rayson, M. J.; Kerridge, A.; Harker, A. H.; Stoneham, A. M. Importance of Quantum Tunneling in Vacancy-Hydrogen Complexes in Diamond. Phys. Rev. Lett. 2005, 95 (10), 105502.

https://doi.org/10.1103/PhysRevLett.95.105502.

\section{Table Of Contents}

Fullerene materials show promise for realizing qubits but current candidates are difficult to synthesise. Here, the preparation of stable and abundant spin-1/2 species from spin-active azafullerene $\left(\mathrm{C}_{59} \mathrm{~N}^{\bullet}\right)$ supramolecularly hosted in [10]cycloparaphenylene ([10]CPP) nanohoops in the solid state is presented. Their operation as stable qubits is demonstrated, with additional possibility of qubit wiring via intermediate polymerized spin-redistributed states.

Y. Tanuma, A.Stergiou, A. Bužan Bobnar, M. Gaboardi, J. Rio, J. Volkmann, H. A. Wegner, N. Tagmatarchis, C. P. Ewels, * D. Arčon*

Robust coherent spin centers from stable azafullerene radicals entrapped in cycloparaphenylene rings 


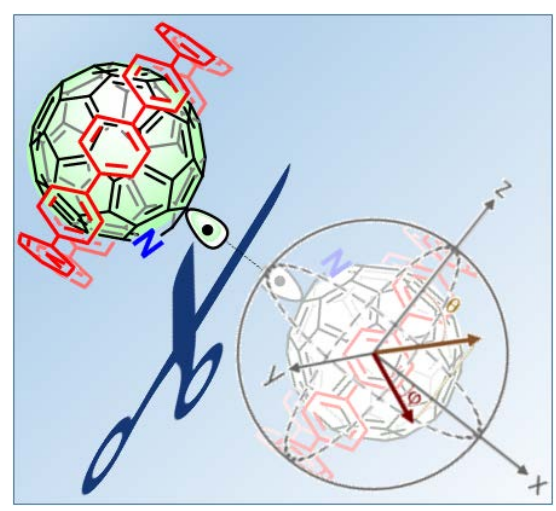

